\title{
Circulating miR-483-3p and miR-21 is highly expressed in plasma of pancreatic cancer
}

\author{
MAKOTO ABUE $^{1}$, MISA YOKOYAMA $^{1}$, RIE SHIBUYA $^{1}$, KEIICHI TAMAI $^{2}$, KAZUNORI YAMAGUCHI $^{3}$, \\ IKURO SATO ${ }^{4}$, NOBUYUKI TANAKA ${ }^{2}$, SHIN HAMADA ${ }^{5}$, TOORU SHIMOSEGAWA ${ }^{5}$, \\ KAZUO SUGAMURA ${ }^{3}$ and KENNICHI SATOH ${ }^{1}$

\begin{abstract}
Divisions of ${ }^{1}$ Cancer Stem Cell,${ }^{2}$ Cancer Biology and Therapeutics, and ${ }^{3}$ Molecular and Cellular Oncology, Miyagi Cancer Center Research Institute; ${ }^{4}$ Department of Pathology, Miyagi Cancer Center, Medeshima Siote, Natori, Miyagi 981-1293; ${ }^{5}$ Division of Gastroenterology, Tohoku University Graduate School of Medicine, Aoba-ku, Sendai, Miyagi 980-8574, Japan
\end{abstract}

Received September 11, 2014; Accepted October 23, 2014

DOI: 10.3892/ijo.2014.2743

\begin{abstract}
Several recent studies have revealed that microRNAs (miRNAs) have a role in carcinogenesis and cancer development, and that it is stably detectable in plasma/serum. The aim of this study was to test whether miR-483-3p as well as miR-21 could be plasma biomarkers for PDAC. The plasma samples were obtained from three groups including 32 pancreatic ductal adenocarcinoma (PDAC) patients, 12 patients with intraductal papillary mucinous neoplasm (IPMN) patients and 30 healthy controls (HC). We evaluated the plasma miR-483-3p and miR-21 expression level by quantitative RT-PCR. We compared the differences in the plasma level of these miRNAs among the three groups, and investigated the relevance of their plasma expression level to the clinical factors in PDAC. The expressions of miR-483-3p and miR-21 were detected in all examined plasma samples. The plasma expression levels of these miRNAs were significantly higher in PDAC compared to $\mathrm{HC}(\mathrm{P}<0.01)$. The plasma miR-483-3p expression was significantly higher in PDAC patients than IPMN patients $(\mathrm{P}<0.05)$. The plasma miR-21 level was associated with advanced stage $(\mathrm{P}<0.05)$, metastasis to lymph node and liver $(\mathrm{P}<0.01)$, and shorter survival $(\mathrm{P}<0.01)$ of the PDAC patients. Together, these findings suggest that measurement of the plasma miR-483-3p level is useful for discriminating PDAC from IPMN, and that the plasma miR-21 level predicts outcome of PDAC patients.
\end{abstract}

Correspondence to: Dr Kennichi Satoh, Division of Cancer Stem Cell, Miyagi Cancer Center Research Institute, 47-1 Nodayama, Medeshima Siote, Natori, Miyagi 981-1293, Japan

E-mail: ksatoh-gi@umin.ac.jp

Key words: plasma, miR-483-3p, miR-21, pancreatic cancer, biomarker

\section{Introduction}

Pancreatic ductal adenocarcinoma (PDAC) is the fifth leading cause of cancer death in Japan (1). This disease usually shows a poor prognosis because of the rapid progression and development of distant metastasis by the time of diagnosis $(2,3)$. In addition, this tumor is resistant to conventional chemotherapy and radiation therapy. Effective technologies such as positron emission tomography (PET) and endoscopic ultrasound-guided fine needle aspiration-biopsy (EUS-FNAB) can improve the detection of PDAC. However, the availability of these methods is restricted due to their high costs or difficulty of technique. On the other hand, the serum levels of carcinoembryonic antigen (CEA) and carbohydrate antigen 19-9 (CA19-9) have been used as useful markers for the presence of PDAC (4). However, their sensitivity and specificity for the early detection of PDAC is not sufficient. Therefore, an easy and accurate method for the detection of PDAC is required to improve its poor prognosis.

MicroRNAs (miRNAs) are small non-coding RNAs composed of 18-25 nucleotides that regulate the translation of specific genes through binding to the 3'-untranslated regions (UTRs) of their target mRNAs. Since first discovered by Lee et al in 1993 (5), accumulating evidence has revealed that miRNAs play important roles in the generation or development of various cancers. In PDAC tissues, a number of studies have identified multiple aberrantly expressed miRNAs, including miR-21 (6-9), miR-155 (7-9), miR-146a (10), miR-196a (11), miR-196b (12), miR-200a/b/c (13-15), miR-221 and miR-222 $(7,16)$. Interestingly, miRNAs are stably detectable in the plasma/serum since they are protected from RNase activity (17-20) by microvesicles like exosomes (21-23), forming protein complexes with Ago2 (24), and lipoprotein complexes (25). The stability of miRNAs in body fluids suggests that circulating miRNAs could be useful diagnostic markers in various cancers. Recently, it has been reported that increased or decreased expression of circulating miRNAs, such as miR-18a, miR-21, miR-155, miR-196a, and miR-200a/b, miR-210, miR-221, are correlated with the development of 
PDAC (20,26-29) (Table I). Among these miRNAs, the correlation between tumor progression and the expression of miR-21 has been well documented in PDAC patients (26,30-37).

We previously performed comprehensive analysis of miRNA expression profiles in PDAC and non-invasive intraductal papillary-mucinous neoplasm (IPMN) (GSE29542) to explore the miRNAs involved in the invasive growth of PDAC. We identified miR-126 and miR-197 as differentially expressed miRNAs in PDAC and revealed that these miRNAs contributed to the invasion of PDAC cells $(38,39)$. We also found miR-483-3p as one of the upregulated miRNAs along with miR-21 and-197 in PDAC by re-reviewing this comprehensive analysis. miR-483-3p, which is located within intron 2 of the IGF2 locus, is overexpressed in a variety of tumors such as malignant mesothelioma, Wilms' tumor tissues, colon, breast and liver cancers $(40,41)$. In addition, the expression of miR-483-3p is strongly enhanced in PDAC tissues and suppresses the expression of DPC4/Smad4 (42). However, little is known about the plasma expression of miR-483-3p and its association with the clinicopathological features in PDAC patients. Therefore, we tested whether evaluation of the plasma miR-483-3p level would be useful for detecting PDAC, and investigated its possible use as a biomarker by comparing it with plasma miR-21 and conventional tumor marker (CEA and CA19-9) levels.

\section{Materials and methods}

Microdissection from tissue samples. Ten PDAC and 13 IPMN tissue samples were obtained from patients who underwent surgical resection in Miyagi Cancer Center Hospital from 2010 to 2012. Histologic diagnosis of each sample was performed by two pathologists who were not informed about the present study. Histologically, cancer duct cells and non-tumor cells (mixed with acinar cells, inflammatory duct cells and stromal cells) from PDAC tissues $(\mathrm{n}=10)$, and non-invasive IPMN cells (adenoma and carcinoma in situ, $\mathrm{n}=13$ ) were microdissected and subjected to RNA extraction. These paraffin-embedded tissues were cut into $10-\mu \mathrm{m}$ sections and $\sim 10$ sequential regions from the same paraffin block were microdissected using a Leica CIR MIC system (Leica Microsystems, Wetzkar, Germany). Total RNA including miRNA was extracted using the Recover All Total Nucleic Acid Isolation kit (Ambion, Austin, TX, USA), according to the manufacturer's instructions.

Patients and blood samples. Between April 2011 and March 2013, the plasma samples of 32 PDAC patients, 30 healthy controls (HC) and 12 IPMN patients were collected at Miyagi Cancer Center. Patient characteristics with respect to age, sex, and stages of disease are described in Table II. The stage of PDAC was assessed according to the Union for International Cancer Control (UICC) Classification (43). All patients were pathologically diagnosed as having PDAC using surgical $(\mathrm{n}=8)$ or biopsy specimens [EUS-FNA $(\mathrm{n}=20)$, endoscopic transpapillary biopsy $(n=3)$ and biopsy from duodenal invasion $(n=1)]$. As a control, plasma samples were collected from $30 \mathrm{HC}$. HC were 22 medical staff members and 8 hospitalized patients with benign disease such as asymptomatic cholecystolithiasis or choledocholithiasis. They underwent medical examinations and did not have any pancreatic disease. All IPMN patients were the branch duct type of IPMNs (BD-IPMN). In this study, the definition of BD- IPMN was based on imaging as a grapelike multilocular cystic lesion without mural nodules communicating with the MPD by computed tomography (CT), magnetic resonance cholangiopancreatography (MRCP), endoscopic ultrasonography (EUS), and/or endoscopic retrograde cholangiopancreatography (ERCP).

Blood plasma samples and RNA extraction. Blood samples were collected from patients and controls in sodium EDTA-Na tubes and were immediately centrifuged at $3500 \mathrm{rpm}$ for $10 \mathrm{~min}$ at room temperature. Then the plasma supernatant was collected and stored at $-80^{\circ} \mathrm{C}$ until further analysis. Total RNA containing small RNA was extracted from $300 \mu$ plasma samples using a mirVana PARIS kit (Ambion), undiluted into $100 \mu \mathrm{l}$ of preheated $\left(95^{\circ} \mathrm{C}\right)$ elution solution according to the manufacturer's instructions. To normalize sample-to-sample variation in the RNA isolation step, $2 \mu \mathrm{l}$ of synthetic C.elegans miR-39, which lacks sequence homology to human miRNAs, was added to each denatured sample.

The detection of miRNAs. The amount of miRNAs was quantified in duplicate via quantitative RT-PCR using the human TaqMan MicroRNA Assay kit (Applied Biosystems, Foster City, CA, USA). The reverse transcription reaction was carried out with a TaqMan MicroRNA Reverse Transcription kit (Applied Biosystems) in $15 \mu \mathrm{l}$ containing $5 \mu \mathrm{l}$ of RNA extract, $0.15 \mu \mathrm{l}$ of $100 \mathrm{mM}$ dNTPs, $1.5 \mu \mathrm{l}$ of $10 \mathrm{X}$ reverse transcriptase buffer, $1 \mu 1$ of Multiscribe reverse transcriptase $(50 \mathrm{U} / \mu 1)$, $0.19 \mu \mathrm{l}$ of RNase inhibitor $(20 \mathrm{U} / \mu \mathrm{l}), 3 \mu \mathrm{l}$ of gene-specific primer, and $4.16 \mu \mathrm{l}$ of Nuclease-free water. For the synthesis of cDNA, reaction mixtures were incubated at $16^{\circ} \mathrm{C}$ for $30 \mathrm{~min}$, at $42^{\circ} \mathrm{C}$ for $30 \mathrm{~min}$, and at $85^{\circ} \mathrm{C}$ for $5 \mathrm{~min}$, and then held at $4^{\circ} \mathrm{C}$. Next, $15 \mu \mathrm{l}$ of cDNA solution was amplified with $25 \mu \mathrm{l}$ of TaqMan 2X Universal PCR Master Mix with no AmpErase UNG (Applied Biosystems), $2.5 \mu 1$ of gene-specific primers/ probe, and $7.5 \mu \mathrm{l}$ of nuclease-free water in a final volume of $50 \mu \mathrm{l}$. Quantitative RT-PCR was run on a LightCycler ${ }^{\circledR} 480$ real-time PCR system (Roche), and reaction mixtures were incubated at $95^{\circ} \mathrm{C}$ for $10 \mathrm{~min}$, followed by 40 cycles of $95^{\circ} \mathrm{C}$ for $15 \mathrm{sec}$, and $60^{\circ} \mathrm{C}$ for $1 \mathrm{~min}$. Cycle threshold $(\mathrm{Ct})$ values were calculated with the LightCycler 480 for Software Version 1.5 (Roche). The relative expression of the mature miRNAs was calculated using the comparative CT $\left(2^{-2 \Delta \Delta t}\right)$ method with miR-16 and RNU6B for plasma and tissue samples, respectively, as the endogenous control to normalize the data $(44,45)$.

Statistical analysis. Student's t-test or Mann-Whitney U test was used to evaluate differences in the miRNA expression between PDAC or IPMN cases and controls. Receiver operating characteristic (ROC) curves were constructed and the area under the curve (AUC) was calculated to evaluate the sensitivity and specificity for predicting cases and controls based on the expression of each individual miRNA and their combinations. Fisher's exact test or Pearson's $\chi^{2}$ test was used to determine if there was a significant association between the clinicopathological factors and the relative plasma expression levels of miRNAs. Survival rate was estimated by the Kaplan-Meier method. The difference in survival 
Table I. Experimentally validated circulating miRNA in PDAC.

\begin{tabular}{|c|c|c|}
\hline miRNAs & Authors/(Ref.) & Expression in PDAC \\
\hline $\operatorname{miR}-21$ & $\begin{array}{l}\text { Wang, et al (26), Liu, et al (34), } \\
\text { Ali, et al (35), Liu, et al (36), } \\
\text { Kong, et al (37) }\end{array}$ & Upregulation \\
\hline $\operatorname{miR}-210$ & $\begin{array}{l}\text { Wang, et al (26), Ho, et al (28), } \\
\text { Liu, et al (36) }\end{array}$ & Upregulation \\
\hline $\operatorname{miR}-155$ & Wang, et al (26), Kon, et al (37) & Upregulation \\
\hline miR-196a & $\begin{array}{l}\text { Wang, et al (26), Liu, et al (36), } \\
\text { Kong, et al (37) }\end{array}$ & Upregulation \\
\hline miR-221 & Kawaguchi, et al (29), Ali, et al (35) & Upregulation \\
\hline miR-200a, 200b & $\mathrm{Li}$, et al (14) & Upregulation \\
\hline $\operatorname{miR}-18 \mathrm{a}$ & Morimura, et al (27) & Upregulation \\
\hline miR-181a,181b & Liu, et al (36) & Upregulation \\
\hline miR-20a, 24, 25, 99a, 185, 191 & Liu, et al (34) & Upregulation \\
\hline miR-1290, 24, 134, 378, 146a, 484, -628-3p, 1825 & $\mathrm{Li}$, et al (53) & Upregulation \\
\hline let-7d, miR-146a & Ali, et al (35) & Downregulation \\
\hline
\end{tabular}

Table II. The characteristics of PDAC patients, IPMN patients and healthy controls (HC).

\begin{tabular}{lccc}
\hline & $\begin{array}{c}\text { PDAC } \\
(\mathrm{N}=32)\end{array}$ & $\begin{array}{c}\text { IPMN } \\
(\mathrm{N}=12)\end{array}$ & $\begin{array}{c}\mathrm{HC} \\
(\mathrm{N}=30)\end{array}$ \\
\hline Age & & & \\
Mean \pm SD & $70.6 \pm 8.7$ & $74.6 \pm 8.8$ & $44.5 \pm 19.1$ \\
(range) & $(48-89)$ & $(61-89)$ & $(21-85)$ \\
Gender & & & \\
Male & 22 & 6 & 11 \\
Female & 10 & 6 & 19 \\
Stage $^{\mathrm{a}}$ & I/II/III/IV & $1 / 8 / 10 / 13$ & \\
\hline
\end{tabular}

${ }^{a}$ UICC classification.

rates between the groups was tested for significance using the log-rank test. The overall survival was defined as the period from the date of diagnosis for PDAC to the date of death or last follow-up. All tests of statistical significance were two-sided. A P-value of $<0.05$ was considered statistically significant. All statistical analyses were done using Excel 2010 software.

Ethics. The Institutional Review Board of the Miyagi Cancer Center (MCC) approved this study protocol, and written informed consent was obtained from each patient (permit MCC-24-38).

\section{Results}

The expression of miR-483-3p as well as miR-21 is enhanced in PDAC cells compared to IPMN and normal pancreatic cells. To validate the results of the previous comprehensive analysis, we first examined the miR-483-3p and miR-21 expression levels in a new series of PDAC and IPMN tissues. The results for these miRNAs, normalized with RNU6B are shown in Fig. 1. In microdissected PDAC cells, the mean level of miR-483-3p expression was $19.36 \pm 5.23$ [miR-483-3p/RNU6B, mean \pm standard error (SE)], and this expression level was significantly higher than that of non-tumor cells $(3.26 \pm 1.22, \mathrm{P}=0.0015)$. The miR-483-3p expression in PDAC cells was also significantly higher than in IPMN lesions $(4.07 \pm 0.75, \mathrm{P}=0.0010)$. Although there was no statistically significant difference between IPMN cells and non-tumor cells, the miR-483-3p level tended to be slightly higher in the IPMN samples than in the normal tissue, suggesting that the increased expression of miR-483-3p occurs in the transition from benign to malignant. Similarly, the expression of miR-21 was significantly higher in PDAC [23150.61 \pm 4493.76 (miR-21/RNU6B, mean \pm SE)] than IPMN $(1413.05 \pm 319.18, \mathrm{P}=0.0001)$ and non-tumor cells $(580.45 \pm 109.06, \mathrm{P}=0.0002)$. On the other hand, miR-21 expression was significantly higher in IPMN compared to non-tumor cells, suggesting that miR-21 contributes to an early step of pancreatic tumorigenesis.

Plasma expression level of miR-483-3p and -21 is increased in PDAC patient. Next, we hypothesized that higher miR-483-3p expression in primary carcinoma cells would influence the plasma levels of miR-483-3p in PDAC patients. To determine the quantities of plasma miRNAs, we used cell-miR-39 as 
A
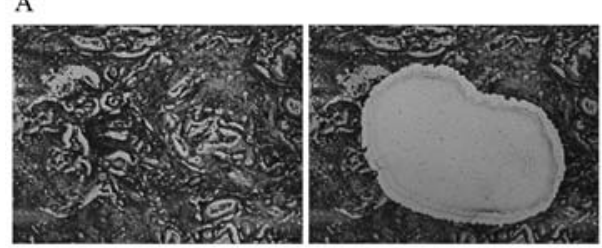

$\mathrm{C}$

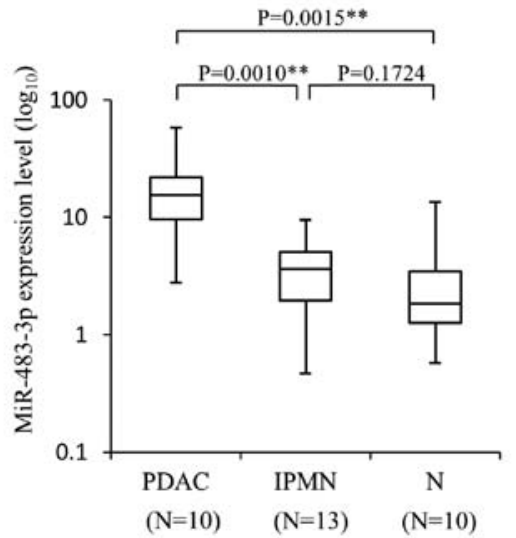

B

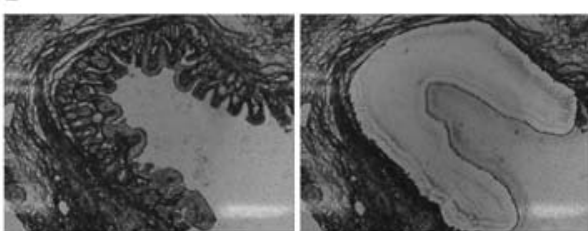

D

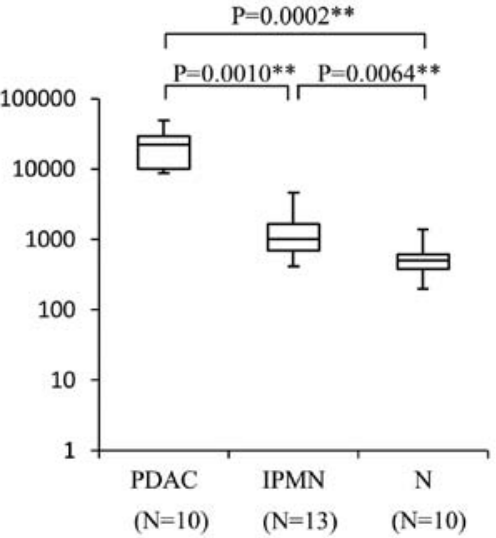

Figure 1. The expression of miR-483-3p and miR-21 in microdissected lesions. MicroRNA extraction was carried out from the microdissected lesions. Neoplastic lesions stained with hematoxylin [left panels in (A) and (B)] were selectively dissected [right panels in (A) and (B)] using a Leica CIR MIC system and recovered in lysis buffer. (A) PDAC tissue; (B) IPMN tissue. (C) The miRNA-483-3p and (D) miR-21 expression levels were detected by quantitative RT-PCR. Boxes represent the interquartile range and the line indicates the median value. Whiskers indicate maximum and minimum values. RNU6B was used for normalization. The miR-483-3p and miR-21 expression levels are significantly higher in PDAC tissue than in non-cancer tissue and IPMN tissue. Mann-Whitney U test was used to evaluate statistical significance. $\mathrm{N}$, non-cancer tissue. ${ }^{* *} \mathrm{P}<0.01$.
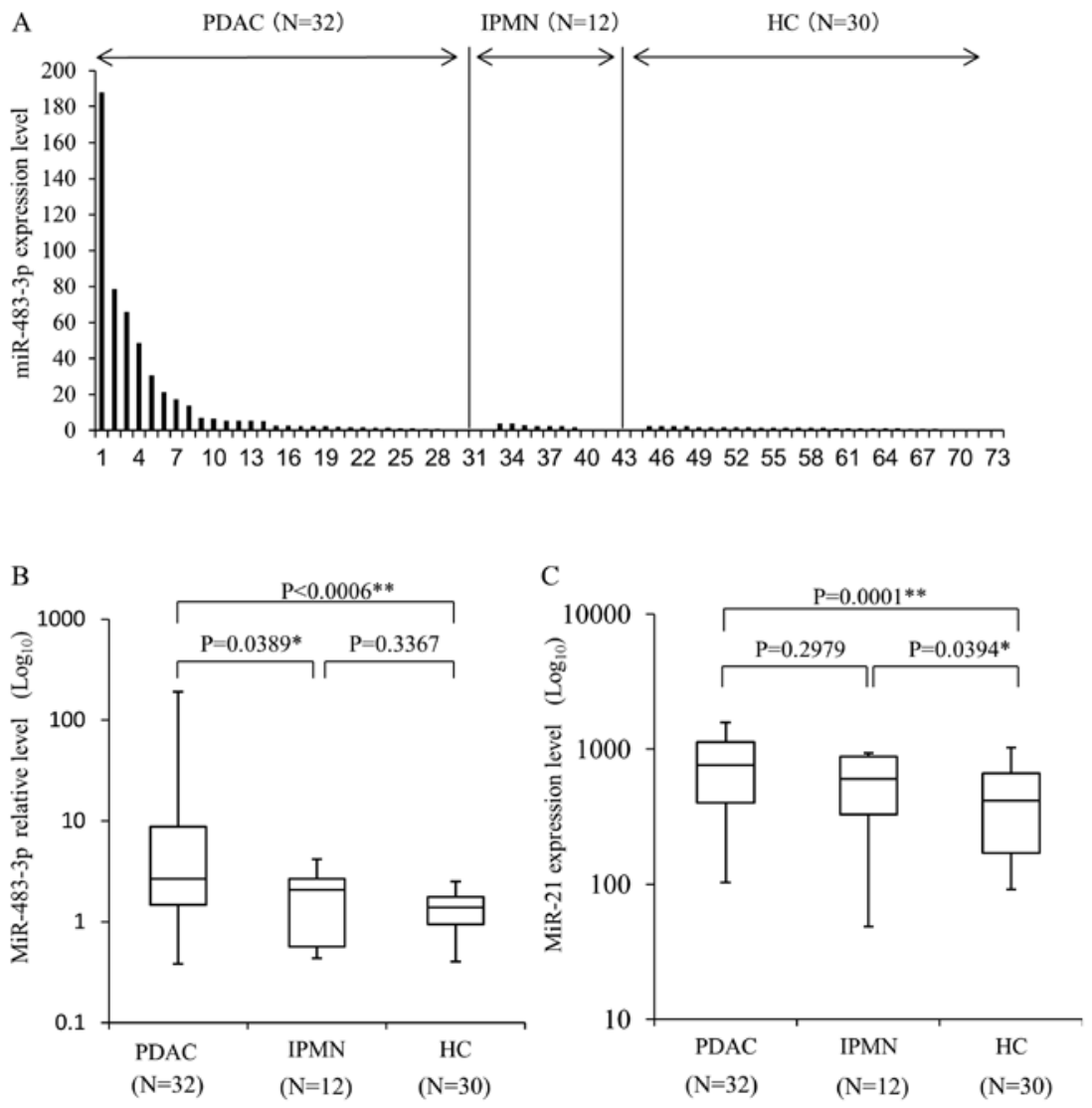

Figure 2. The expression of miR-483-3p and miR-21 in plasma samples. (A) The distribution of the plasma miR-483-3p expression level in all samples. (B) The miR-483-3p expression level in PDAC, IPMN and normal plasma samples. Boxes represent the interquartile range and the line indicates the median value. Whiskers indicate maximum and minimum values. miR-16 was used for normalization. The miR-483-3p expression level in plasma is significantly higher in PDAC than HC and IPMN group, while no significant difference is found between IPMN and the HC group. Mann-Whitney U test was used to evaluate statistical significance. (C) The plasma miR-21 expression level in PDAC, IPMN and HC. The plasma miR-21 level is significantly higher in PDAC than HC group, whereas no significant difference was observed between PDAC and IPMN. ${ }^{*} \mathrm{P}<0.05$ and ${ }^{* * *} \mathrm{P}<0.01$. 

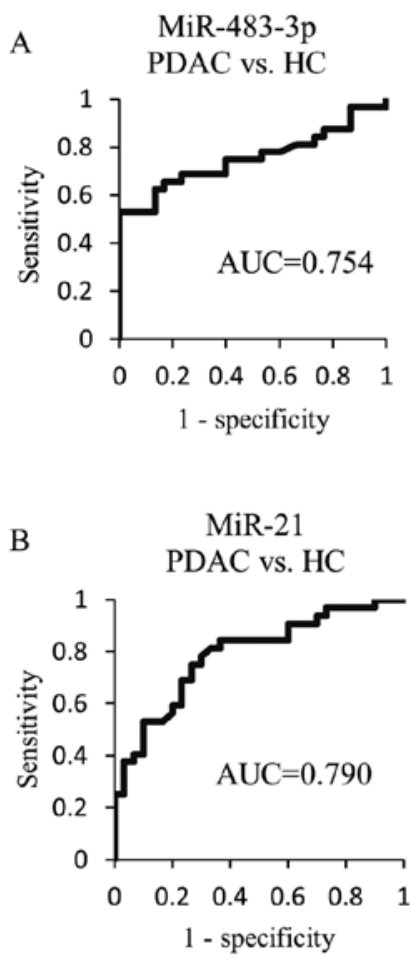

C $\quad \operatorname{miR}-483-3 \mathrm{p} \times \mathrm{miR}-21$ PDAC vs. $\mathrm{HC}+$ IPMN

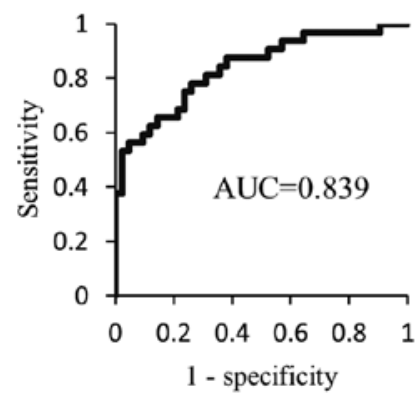

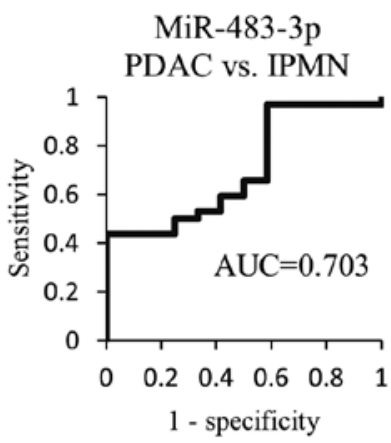

MiR-21 PDAC vs. IPMN

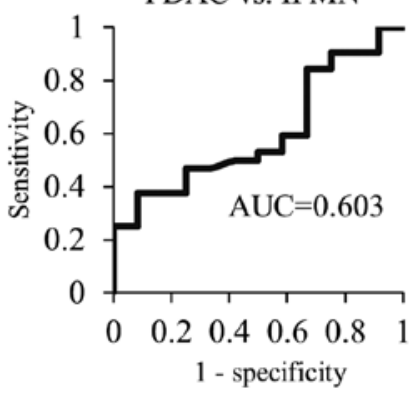

D CEA
PDAC vs. HC + IPMN

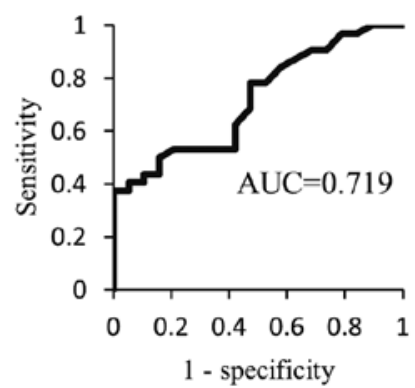

MiR-483-3p

PDAC vs. HC+IPMN

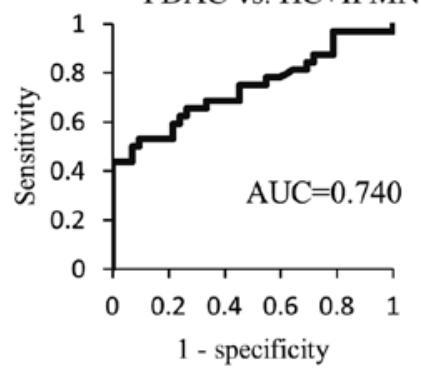

MiR-21

PDAC vs. HC+IPMN

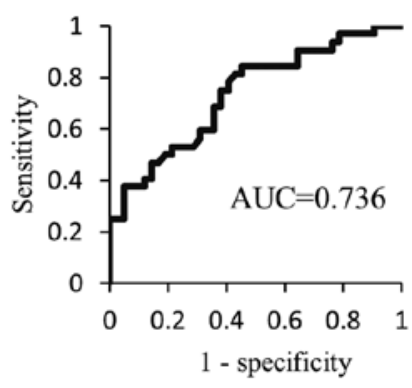

E

CA19-9

PDAC vs. HC+IPMN

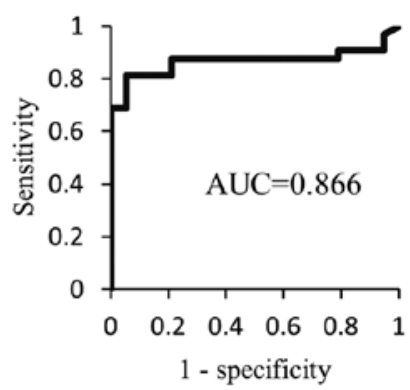

Figure 3. The ROC curve analysis of plasma miRNA and serum tumor marker levels for PDAC diagnosis. (A) The ROC curves for differentiating between PDAC and HC (left), PDAC and IPMN (center), PDAC, and HC and IPMN (right) based on the miR-483-3p plasma level. (B) The ROC curves for differentiating between PDAC and HC (left), PDAC and IPMN (center), PDAC, and HC and IPMN (right) based on the plasma miR-21 level. The ROC curves for differentiating PDAC from IPMN and HC based on the combination of the miR-483-3p and miR-21 plasma levels (C), serum CEA (D) and serum CA19-9 level (E).

internal control miRNAs, and miR-16 was used for the normalization. Plasma miR-483-3p expression was calculated from the comparative $\mathrm{Ct}$ method by quantitative RT-PCR. Using this assay, circulating miR-483-3p was detectable in all plasma samples from 32 PDAC patients, $30 \mathrm{HC}$ and 12 IPMN patients (Fig. 2A). The mean level of plasma miR-483-3p expression in the PDAC patients $[16.50 \pm 6.49(\mathrm{miR}-483-3 \mathrm{p} / \mathrm{miR}-16$, mean $\pm \mathrm{SE})]$ was significantly higher than in $\mathrm{HC}(1.37 \pm 0.11, \mathrm{P}=0.0006)$ and IPMN patients $(1.90 \pm 0.39, \mathrm{P}=0.0389)$ (Fig. $2 \mathrm{~B})$. We also evaluated the expression level of plasma miR-21, which was previously shown to be overexpressed in the plasma and tissue of PDAC (26,36-43). The mean expression level of plasma miR-21 (Fig. 2C) was significantly higher in the PDAC group [765.25 $\pm 64.6(\mathrm{miR}-21 / \mathrm{miR}-16 \pm \mathrm{SE})]$ than HC $(414.54 \pm 44.7$, $\mathrm{P}=0.0001)$. The plasma expression level of miR-21 in IPMN patients was also higher than in $\mathrm{HC}(\mathrm{P}=0.0394)$, and no differ- ences were seen between PDAC and IPMN ( $\mathrm{P}=0.2979)$. While no difference was observed in the plasma miR-21 expression level between PDAC and IPMN patients, miR-483-3p expression in the plasma of PDAC patients was significantly higher than in that of IPMN patients. This result indicates that plasma miR-483-3p measurement has the potential to distinguish PDAC not only from healthy individuals, but also from the patient of IPMN.

The utility of measuring miR-483-3p and -21 in plasma for discriminating PDAC from healthy controls and/or IPMN patients. To apply the expression value of plasma miRNAs for the diagnosis of PDAC, we then analyzed the ROC curves. The ROC curves for differentiating between PDAC and $\mathrm{HC}$, and/or IPMN based on the expression level of miRNAs (miR-483-3p or miR-21) are shown in Fig. 3. The AUC of 
Table III. The association of miR-483-3p expression level with clinicopathological factors in PDAC.

\begin{tabular}{ccc}
\hline Low & High & P-value \\
expression & expression & \\
$(\mathrm{N}=18)$ & $(\mathrm{N}=14)$ & \\
\hline
\end{tabular}

Age

$<70$

$\geq 70$

8

10

8

0.476

Gender

Male

Female

$$
12
$$

6

10

0.773

Diabetes mellitus

Yes

9

9

8

6

0.688

Location

Head

Body-tail

$$
7
$$

7

11

7

Tumor size (mm)

Mean \pm SD

$45.6 \pm 22.0$

$38.3 \pm 12.6$

0.424

T classification $^{\mathrm{a}}$

T1-T2

7

11

8

6

$\mathrm{N}_{\text {classification }}{ }^{\mathrm{a}}$

Yes

No

$$
5
$$

$$
4
$$

10

0.960

Liver metastasis

$\begin{array}{lrr}\text { Yes } & 5 & 4 \\ \text { No } & 13 & 10\end{array}$

Ascites

$\begin{array}{lrr}\text { Yes } & 2 & 2 \\ \text { No } & 16 & 12\end{array}$

Stage $^{\mathrm{a}}$

I-III

IV

10

8

9

5

0.305

0.530

Head

Body-tail

Tumor size (mm)

Mean \pm SD

$$
38.8 \pm 15.8
$$

$50.3 \pm 22.5$

0.137

$\mathrm{T}_{\text {classification }}{ }^{\mathrm{a}}$

\section{T1-T2}

T3-T4

$\mathrm{N}_{\text {classification }}{ }^{\mathrm{a}}$

12

10

3

7

0.960

Operation

\begin{tabular}{lrrr} 
Yes & 3 & 5 & 0.217 \\
No & 15 & 9 & \\
\hline
\end{tabular}

${ }^{\mathrm{a} U I C C}$ classification.

miR-483-3p (0.754) was slightly lower than that of miR-21 (0.790) for differentiating PDAC from HC. On the other hand, for discriminating PDAC from IPMN patients, the AUC of miR-483-3p (0.703) was higher than that of miR-21 (0.603), In addition, the AUC value based on the expression level of plasma miR-483-3p (0.740) and miR-21 (0.736) to distinguish PDAC patients from non-cancer individuals (HC and
Yes

No

Liver metastasis

Yes

No

Ascites

Yes

No

Stage $^{\mathrm{a}}$

I-III

IV

Operation

Yes

No$$
3
$$

6

4

$0.007^{c}$

$$
2
$$

7

3

$<0.001^{\mathrm{c}}$

${ }^{\mathrm{a} U I C C}$ classification. ${ }^{\mathrm{b}} \mathrm{P}<0.05 ;{ }^{\mathrm{c}} \mathrm{P}<0.01$.

IPMN) was similar. Consequently, we compared the ability of measuring the plasma miR-483-3p and miR-21 to predict PDAC with that of the conventional tumor markers CEA and CA19-9. The respective AUC values of plasma miR-483-3p and miR-21 were higher than that of CEA (0.719), but lower than that of CA19-9 (0.866). Interestingly, the AUC (0.839) from the ROC curves for the combination of miR-483-3p 

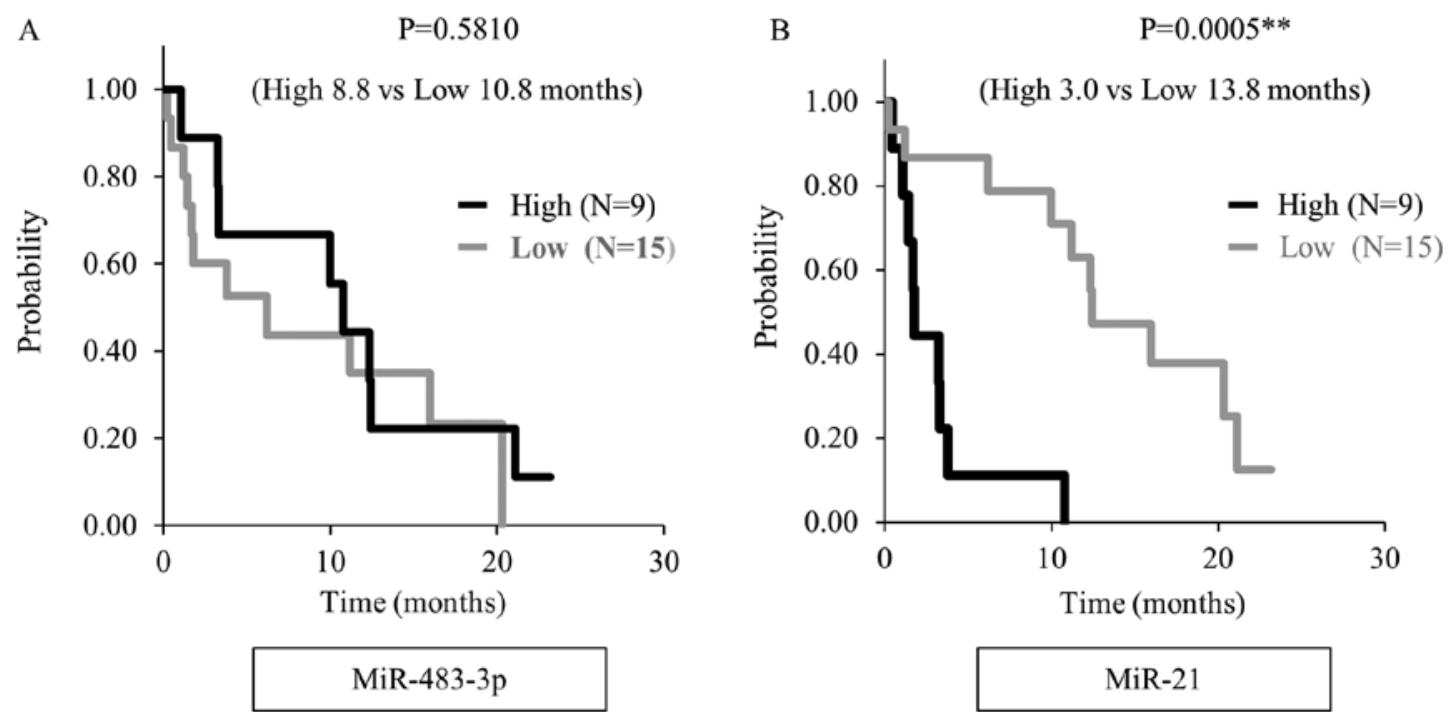

Figure 4. The correlation between plasma miRNA expression and overall survival (OS) of PDAC patients. Kaplan-Meier overall survival curve in 24 PDAC patients who were excluded based on their plasma miRNA expression. (A) There was no significant relationship between the plasma miR-483-3p level and overall survival. (B) The prognosis for PDAC patients was worse in those with high expression of plasma miR-21. ${ }^{* *} \mathrm{P}<0.01$.
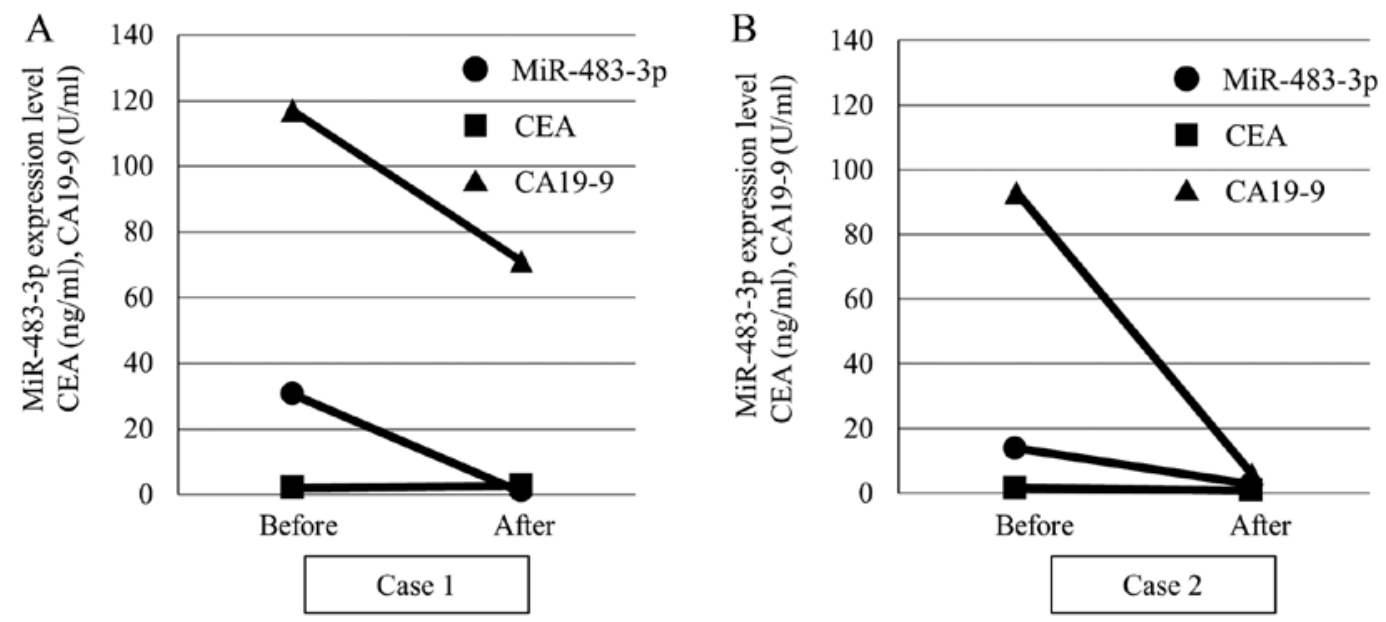

Figure 5. The plasma expression level of miR-483-3p and tumor markers before and after surgery. The plasma miR-483-3p level was drastically decreased after surgery in two PDAC patients: A, case 1 and B, case 2. Both cases were diagnosed as pancreatic adenocarcinoma and were classified as stage IIA (T3NOM0) using the UICC classification.

and miR-21 was similar to that of CA19-9 for discriminating patients with PDAC from non-cancer individuals.

The correlation between plasma miRNAs and clinicopathological factors. Finally, we evaluated the correlation between the plasma level of miRNAs and clinicopathological factors in 32 PDAC patients (Table III). We judged the miR-483-3p level as 'high expression' when it was higher than a cut-off value of 5. There was no significant relationship between the plasma miR-483-3p level and clinical factors with overall survival. The plasma miR-21 expression level was associated with advanced stage $(\mathrm{P}=0.023)$, metastasis to lymph node $(\mathrm{P}=0.007)$ and liver $(\mathrm{P}<0.001)$ when the cut-off value was defined as 850 , as shown in Table IV. Moreover, overall survival was significant shorter in the high miR-21 expression group than in the low expression group when we analyzed by the Kaplan-Meier method in
24 unresectable PDAC patients, (3.0 months vs 13.8 months, $\mathrm{P}<0.001)$ (Fig. 4).

Plasma miR-483-3p expression level is decreased after resection of PDAC. Since the plasma miR-21 level was not increased in operable patients (Table IV), we evaluated alterations in the plasma miR-483-3p level alone after resection of the tumor tissues. Fig. 5 shows representative cases of the plasma expression level of miR-483-3p and tumor markers before and after surgery. Case 1 was a 70-year-old female and case 2 was a 62-year-old female. In both cases, pancreaticoduodenectomy was performed for the diagnosis of pancreatic head cancer. By histological examination, the diagnosis was pancreatic adenocarcinoma and the classification stage IIA (T3NOM0) using UICC classification. As expected, a marked reduction of the plasma miR-483-3p expression as well as the CA19-9 
value was observed after surgery, indicating that the expression of miR-483-3p was strongly associated with the presence of PDAC.

\section{Discussion}

MiRNAs have been shown to play important roles in the tumorigenesis and/or development of various cancers. Since it exists stably in the plasma/serum, miRNA appears promising as a new biomarker for the diagnosis and treatment of cancer. In PDAC patients, numerous miRNAs including miR-21, miR-155, miR-196a and miR-210 have been detected in the plasma/serum, and have been suggested to be useful biomarkers for the diagnosis $(6,7,9,26)$. In the present study, we examined the expression of miR-483-3p as well as miR-21 in the plasma of PDAC patient to assess whether these miRNAs would be useful markers for the detection of PDAC and for predicting the clinical status of this tumor. We clearly demonstrated for the first time that the plasma miR-483-3p level was significantly higher in PDAC patients compared to those of $\mathrm{HC}$ and IPMN patients and that the AUC value of miR-483-3p for discriminating PDAC from IPMN was higher than that of miR-21. miR-483-3p has been shown to promote cell proliferation through downregulation of its target gene, Smad4 in PDAC cells (42). Reportedly, the loss of Smad4 expression is frequently observed from the late stage of carcinogenesis of PDAC, but infrequently in IPMN $(46,47)$. Taken together with our finding that miR-483-3p is expressed specifically in PDAC patients without showing a relationship to the clinical features, this miRNA may play a pivotal role in the carcinogenesis rather than the development of PDAC through reducing Smad4 expression.

Recent studies revealed that BD-IPMN is a risk factor for PDAC since the occurrence of PDAC during the follow-up period for BD-IPMN patients was not infrequent (48-50). To date, there are no highly sensitive methods to predict the occurrence of PDAC in IPMN patients. However, worsening diabetes mellitus and abnormal serum CA19-9 levels have been demonstrated to be useful to predict the development of PDAC in BD-IPMN patients $(51,52)$, and the sensitivity of CA19-9 to predict concomitant PDAC was shown to be $45 \%$ (51). In the present study, the sensitivity of the plasma miR-483-3p level to differentiate PDAC from IPMN was $43.8 \%$, similar to that of CA19-9, indicating that the evaluation of this miRNA in plasma could also be a useful tool for detecting the occurrence of PDAC in IPMN patients. The reduction of the plasma miR-483-3p level by surgical resection of PDAC may partially support this idea.

Overexpression of plasma miR-21 was demonstrated in PDAC and was correlated with a poor prognosis in this tumor (26,35-37). As had been shown in previous studies, we also demonstrated that the plasma miR-21 level was increased in PDAC patients compared to $\mathrm{HC}$ and associated with poor prognosis in unresectable PDAC patients. In addition, we revealed the relationship between the plasma miR-21 level and liver metastasis in PDAC patients. The involvement of MiR-21 in the metastasis of PDAC cells was also clarified in vitro. Moriyama et al showed that miR-21 precursor transfection promoted proliferation, invasion and gemcitabine resistance in PDAC cells in vitro (30). Moreover, Kadera et al reported that expression of miR-21 in tumor-associated fibroblasts as well as PDAC cells enhanced the metastatic potential (33). These findings suggest that stromal cells as well as metastatic PDAC cells are likely to be the origin of plasma miR-21, and thus high plasma levels of this miRNA are detected in patients with advanced stage PDAC.

Intriguingly, the AUC value for the diagnosis of PDAC was increased to a level similar to that of CA19-9 when the miR-483-3p and miR-21 plasma levels were combined. This is due to mutually independent mechanisms that enhance the expression of the respective miRNAs. As discussed above, miR-483-3p and miR-21 are upregulated in the process of carcinogenesis and development, respectively. Thus, the evaluation of miR-483-3p together with miR-21 would forecast the status in patients with PDAC.

\section{Acknowledgements}

This study was supported by Grants-in-Aid for Scientific Research (KAKENHI) (24591022) to K.S., Pancreas Research Foundation of Japan to M.A. and The Naito Foundation to K.S.

\section{References}

1. Hirata K, Egawa S, Kimura Y, et al: Current status of surgery for pancreatic cancer. Dig Surg 24: 137-147, 2007.

2. Siegel R, Ma J, Zou Z and Jemal A: Cancer statistics, 2014. CA Cancer J Clin 64: 9-29, 2014.

3. Vincent A, Herman J, Schulick R, Hruban RH and Goggins M: Pancreatic cancer. Lancet 378: 607-620, 2011.

4. Satake K, Kanazawa G, Kho I, Chung YS and Umeyama K: A clinical evaluation of carbohydrate antigen 19-9 and carcinoembryonic antigen in patients with pancreatic carcinoma. J Surg Oncol 29: 15-21, 1985

5. Lee RC, Feinbaum RL and Ambros V: The C.elegans heterochronic gene lin-4 encodes small RNAs with antisense complementarity to lin-14. Cell 75: 843-854, 1993.

6. Dillhoff M, Liu J, Frankel W, Croce C and Bloomston M: MicroRNA-21 is overexpressed in pancreatic cancer and a potential predictor of survival. J Gastrointest Surg 12: 2171-2176, 2008.

7. Bloomston M, Frankel WL, Petrocca F, et al: MicroRNA expression patterns to differentiate pancreatic adenocarcinoma from normal pancreas and chronic pancreatitis. JAMA 297: 1901-1908, 2007.

8. Habbe N, Koorstra JB, Mendell JT, et al: MicroRNA miR-155 is a biomarker of early pancreatic neoplasia. Cancer Biol Ther 8: 340-346, 2009.

9. Ryu JK, Hong SM, Karikari CA, Hruban RH, Goggins MG and Maitra A: Aberrant microRNA-155 expression is an early event in the multistep progression of pancreatic adenocarcinoma. Pancreatology 10: 66-73, 2010.

10. Li Y, Vandenboom TG II, Wang Z, Kong D, Ali S, Philip PA and Sarkar FH: miR-146a suppresses invasion of pancreatic cancer cells. Cancer Res 70: 1486-1495, 2010.

11. Zhang Y, Li M, Wang H, Fisher WE, Lin PH, Yao Q and Chen C: Profiling of 95 microRNAs in pancreatic cancer cell lines and surgical specimens by real-time PCR analysis. World J Surg 33: 698-709, 2009.

12. Yu J, Li A, Hong SM, Hruban RH and Goggins M: MicroRNA alterations of pancreatic intraepithelial neoplasias. Clin Cancer Res 18: 981-992, 2012.

13. Kent OA, Mullendore M, Wentzel EA, et al: A resource for analysis of microRNA expression and function in pancreatic ductal adenocarcinoma cells. Cancer Biol Ther 8: 2013-2024, 2009.

14. Li A, Omura N, Hong SM, et al: Pancreatic cancers epigenetically silence SIP1 and hypomethylate and overexpress miR-200a/200b in association with elevated circulating miR-200a and miR-200b levels. Cancer Res 70: 5226-5237, 2010 . 
15. Yu J, Ohuchida K, Mizumoto K, et al: MicroRNA, hsa-miR-200c, is an independent prognostic factor in pancreatic cancer and its upregulation inhibits pancreatic cancer invasion but increases cell proliferation. Mol Cancer 9: 169, 2010.

16. Lee EJ, Gusev Y, Jiang J, et al: Expression profiling identifies microRNA signature in pancreatic cancer. Int J Cancer 120 1046-1054, 2007.

17. Calin GA and Croce CM: MicroRNA signatures in human cancers. Nat Rev Cancer 6: 857-866, 2006.

18. Chen X, Ba Y, Ma L, et al: Characterization of microRNAs in serum: a novel class of biomarkers for diagnosis of cancer and other diseases. Cell Res 18: 997-1006, 2008.

19. Filipowicz W, Bhattacharyya SN and Sonenberg N: Mechanisms of posttranscriptional regulation by microRNAs: are the answers in sight? Nat Rev Genet 9: 102-114, 2008.

20. Mitchell PS, Parkin RK, Kroh EM, et al: Circulating microRNAs as stable blood-based markers for cancer detection. Proc Natl Acad Sci USA 105: 10513-10518, 2008.

21. Hasselmann DO, Rappl G, Tilgen W and Reinhold U: Extracellular tyrosinase mRNA within apoptotic bodies is protected from degradation in human serum. Clin Chem 47 1488-1489, 2001.

22. Cocucci E, Racchetti G and Meldolesi J: Shedding microvesicles: artefacts no more. Trends Cell Biol 19: 43-51, 2009.

23. Kosaka N, Iguchi H, Yoshioka Y, Takeshita F, Matsuki Y and Ochiya T: Secretory mechanisms and intercellular transfer of microRNAs in living cells. J Biol Chem 285: 17442-17452, 2010.

24. Arroyo JD, Chevillet JR, Kroh EM, et al: Argonaute2 complexes carry a population of circulating microRNAs independent of vesicles in human plasma. Proc Natl Acad Sci USA 108: 5003-5008, 2011.

25. Vickers KC, Palmisano BT, Shoucri BM, Shamburek RD and Remaley AT: MicroRNAs are transported in plasma and delivered to recipient cells by high-density lipoproteins. Nat Cell Biol 13: 423-433, 2011.

26. Wang J, Chen J, Chang P, et al: MicroRNAs in plasma of pancreatic ductal adenocarcinoma patients as novel bloodbased biomarkers of disease. Cancer Prev Res (Phila) 2: $807-813,2009$

27. Morimura R, Komatsu S, Ichikawa D, et al: Novel diagnostic value of circulating miR-18a in plasma of patients with pancreatic cancer. Br J Cancer 105: 1733-1740, 2011.

28. Ho AS, Huang X, Cao H, Christman-Skieller C, Bennewith K, Le QT and Koong AC: Circulating miR-210 as a novel hypoxia marker in pancreatic cancer. Transl Oncol 3: 109-113, 2010.

29. Kawaguchi T, Komatsu S, Ichikawa D, et al: Clinical impact of circulating miR-221 in plasma of patients with pancreatic cancer Br J Cancer 108: 361-369, 2013.

30. Moriyama T, Ohuchida K, Mizumoto K, et al: MicroRNA-21 modulates biological functions of pancreatic cancer cells including theirproliferation, invasion, and chemoresistance. Mol Cancer Ther 8: 1067-1074, 2009.

31. Giovannetti E, Funel N, Peters GJ, et al: MicroRNA-21 in pancreatic cancer: correlation with clinical outcome and pharmacologic aspects underlying its role in the modulation of gemcitabine activity. Cancer Res 70: 4528-4538, 2010.

32. Hwang JH, Voortman J, Giovannetti E, et al: Identification of microRNA-21 as a biomarker for chemoresistance and clinical outcome following adjuvant therapy in resectable pancreatic cancer. PLoS One 5: e10630, 2010.

33. Kadera BE, Li L, Toste PA, Wu N, Adams C, Dawson DW and Donahue TR: MicroRNA-21 in pancreatic ductal adenocarcinoma tumor-associated fibroblasts promotes metastasis. PLoS One 8: e71978, 2013.

34. Liu R, Chen X, Du Y, et al: Serum miRNA expression profile as a biomarker in the diagnosis and prognosis of pancreatic cancer. Clin Chem 58: 610-618, 2012.
35. Ali S, Almhanna K, Chen W, Philip PA and Sarkar FH Differentially expressed miRNAs in the plasma may provide a molecular signature for aggressive pancreatic cancer. Am J Transl Res 28: 28-47, 2010.

36. Liu J, Gao J, Du Y, et al: Combination of plasma microRNAs with serum CA19-9 for early detection of pancreatic cancer. Int J Cancer 131: 683-691, 2012

37. Kong X, Du Y, Wang G, et al: Detection of differentially expressed microRNAs in serum of pancreatic ductal adenocarcinoma patients: miR-196a could be a potential marker for poor prognosis. Dig Dis Sci 56: 602-609, 2011.

38. Hamada S, Satoh K, Fujibuchi W, et al: MiR-126 acts as a tumor suppressor in pancreatic cancer cells via the regulation of ADAM9. Mol Cancer Res 10: 3-10, 2012.

39. Hamada S, Satoh K, Miura S, et al: MiR-197 induces epithelialmesenchymal transition in pancreatic cancer cells by targeting p120 catenin. J Cell Physiol 228: 1255-1263, 2013.

40. Veronese, A, Lupini L, Consiqlio J, et al: Oncogenic role of miR-483-3p at the IGF2/483 locus. Cancer Res 70: 3140-3149, 2010.

41. Guled M, Lahti L, Lindholm PM, Salmenkivi K, Bagwan I, Nicholson AG and Knuutila S: CDKN2A, NF2, and JUN are dysregulated among other genes by miRNAs in malignant mesothelioma-A miRNA microarray analysis. Genes Chromosomes Cancer 48: 615-623, 2009.

42. Hao J, Zhang S, Zhou Y, Hu X and Shao C: MicroRNA 483-3p suppresses the expression of DPC4/Smad4 in pancreatic cancer. FEBS Lett 585: 207-213, 2011.

43. Sobin LH, Gospodarowicz MK and Wittekind C (eds): TNM Cassification of Malignant Tumours. 7th edition. Wiley-Blackwell, New York, NY, 2009.

44. Schmittgen TD and Livak KJ: Analyzing real-time PCR data by the comparative C(T) method. Nat Protoc 3: 1101-1108, 2008

45. Liang Y, Ridzon D, Wong L and Chen C: Characterization of microRNA expression profiles in normal human tissues. BMC Genomics 8: 166, 2007.

46. Wilentz RE, Iacobuzio-Donahue CA, Argani P, et al: Loss of expression of Dpc4 in pancreatic intraepithelial neoplasia: evidence that DPC4 inactivation occurs late in neoplastic progression. Cancer Res 60: 2002-2006, 2000.

47. Biankin AV1, Biankin SA, Kench JG, et al: Aberrant p16 (INK4A) and DPC4/Smad4 expression in intraductal papillary mucinous tumours of the pancreas is associated with invasive ductal adenocarcinoma. Gut 50: 861-868, 2002

48. Yamaguchi K1, Ohuchida J, Ohtsuka T, Nakano K and Tanaka M Intraductal papillary-mucinous tumor of the pancreas concomitant with ductal carcinoma of the pancreas. Pancreatology 2: 484-490, 2002

49. Uehara H, Nakaizumi A, Ishikawa O, et al: Development of ductal carcinoma of the pancreas during follow-up of branch duct intraductal papillary mucinous neoplasm of the pancreas. Gut 57: 1561-1565, 2008.

50. Tanno S, Nakano Y, Koizumi K, et al: Pancreatic ductal adenocarcinomas in long-term follow-up patients with branch duct intraductal papillary mucinous neoplasms. Pancreas 39: 36-40, 2010.

51. Ingkakul T1, Sadakari Y, Ienaga J, Satoh N, Takahata S and Tanaka M: Predictors of the presence of concomitant invasive ductal carcinoma in intraductal papillary mucinous neoplasm of the pancreas. Ann Surg 25: 70-75, 2010.

52. Kanno A, Satoh K, Hirota M, et al: Prediction of invasive carcinoma in branch type intraductal pappilary mucinous neoplasms of the pancreas. J Gastroenterol 45: 952-959, 2010

53. Li A1, Yu J, Kim H, Wolfgang CL, Canto MI, Hruban RH and Goggins M: MicroRNA array analysis finds elevated serum miR-1290 accurately distinguishes patients with low-stage pancreatic cancer from healthy and disease controls. Clin Cancer Res 19: 3600-3610, 2013 